\title{
ECOGRAFÍA EN EL DIAGNÓSTICO DE LA INCONTINENCIA URINARIA FEMENINA.
}

Miguel Angel Jiménez Cidre, Luis López-Fando Lavalle, Cristina Quicios Dorado, Cristina de Castro Guerín, Agustín Fraile Poblador y Teodoro Mayayo Dehesa.

Servicio de Urología. Hospital Ramón y Cajal. Madrid. España.

Resumen.- OBJETIVO: El valor de la Ecografía en el estudio de la Incontinencia Urinaria Femenina (IUF) ha sido redefinido en los últimos años

MÉTODOS: Se revisa la literatura sobre el valor de la Ecografía en el estudio de las mujeres con Incontinencia urinaria, fundamentalmente en la ecografía transperineal en la Incontinencia Urinaria de esfuerzo femenina

RESULTADOS: En los últimos años son numerosos los trabajos publicados. La ecografía del TUS tiene poco lugar en la evaluación de la IUF de esfuerzo pura. La ecografía transperineal permite evaluar movilidad del cuello vesical y uretra, grosor de la pared vesical, embudización del cuello, presencia de IUF de esfuerzo, presencia de prolapso de órganos pelvianos (POP), visualización de mallas, ayudar al biofeedback y valorar cambios tras tratamiento quirúrgico.
Miguel Angel Jiménez Cidre

Servivio de Urología

Hospital Ramón y Cajal

Ctra. Colmenar Viejo Km 9, 1

28034 Madrid (España)

mjcidre00@hotmail.com
CONCLUSIONES: Los ultrasonidos y en particular la ECO transperineal o translabial está en proceso de convertirse un método diagnóstico estándar en uroginecología. A ello contribuye su amplia disponibilidad, estandarización de los parámetros y la posibilidad de evaluar no sólo la veiiga, sino también el elevador del ano o los Prolapsos de órganos pelvianos (POP). Permite obtener, de forma no invasiva datos pre y postratamiento

Palabras clave: Incontinencia urinaria femenina. Ecografía. Diagnóstico. Ecografía urológica.

Summary.- OBJECTIVES: The value of ultrasonography for the study of female urinary incontinence has been redefined over the last years.

METHODS: We review the literature about the value of ultrasound in the workup of females with urinary incontinence, mainly transperineal ultrasound for the female stress urinary incontinence (SUI).

RESULTS: Many papers have been published over the last few years. Upper urinary tract ultrasound has not a place in the workup of genuine female SUI. Transperineal ultrasound allows to evaluate the mobility of the bladder neck and urethra, the thickness of the bladder wall, the funnel shape of the bladder neck, the presence of SUI or pelvic organ prolapse (POP), to visualize mesh implants, to help with biofeedback, and to evaluate changes after surgical treatment.

CONCLUSIONS: Ultrasounds in general, and transperineal or translabial ultrasound in particular, are in the process of becoming the standard diagnostic method in urogynecology. Their wide availability, the standardization of parameters, the possibility of evaluating not only the 
bladder but also the levator ani muscle or pelvic organ prolapses (POP) contribute to this fact. It allows to obtain data in a non invasive way before and after therapy.

Keywords: Female urinary incontinence. Ultrasound. Diagnosis. Urological ultrasound.

\section{INTRODUCCIÓN}

El valor de la Ecografía en el estudio de la Incontinencia Urinaria Femenina (IUF) ha sido redefinido en los últimos años. Revisamos la situación actual de su uso. Las indicaciones de evaluación del Tracto Urinario Superior (TUS) en la IUF son escasas. La ecografía abdominal en la evaluación del residuo postmiccional está ampliamente aceptada. La necesidad de poner de manifiesto el defecto anatómico del cuello vesical/uretra en la IUF de esfuerzo hizo que históricamente se desarrollaran estudios radiológicos: Cistouretrografía Miccional Seriada (CUMS) y Cistografía con cadena, pero estas presentan la necesidad de irradiar a la paciente y los inconvenientes añadidos del coste y la dependencia de otros servicios.

La aparición y extensión de la ecografía por diversas vías, su bajo coste, inmediatez, ausencia de irradiación y efectos secundarios, baja invasividad y amplia disponibilidad en los servicios de Urología y Ginecología hicieron que esta ganara adeptos en el estudio morfológico del tracto urinario inferior. Se ha usado la ecografía, en el estudio de la posición del cuello vesical y uretra en la IUF, por 6 vías: abdominal $(9,10)$, transperineal (o translabial) (1), transrectal (23), transvaginal $(4)$, introital $(5,6)$, e intrauretral, con diferentes transductores, y se comentará su utilidad en las siguientes páginas (Figura 1).

En esta revisión nos centraremos fundamentalmente en el uso de la ecografía en el estudio de la IUF de esfuerzo.

\section{Ecografía Abdominal y del Tracto urinario superior y Pelviana en la Incontinencia Urinaria Femenina.}

El uso de la ecografía para el estudio de las estructuras abdominopélvicas data de los años 50, pero la valoración de la vejiga se limitó a la estimación del volumen urinario, la movilidad de la pared vesical, distorsión por las masas pélvicas, y detección de los tumores vesicales (7). La estimación del residuo postmiccional fue el primer uso de la ecografía abdominal y está ampliamente aceptado pues evita la necesidad del sondaje vesical. Además la ecografía abdominal se usa en el estudio de las masas pel- vianas que pueden contribuir a la producción de incontinencia. También pueden detectarse patologías del tracto urinario superior, divertículos vesicales y litiasis.

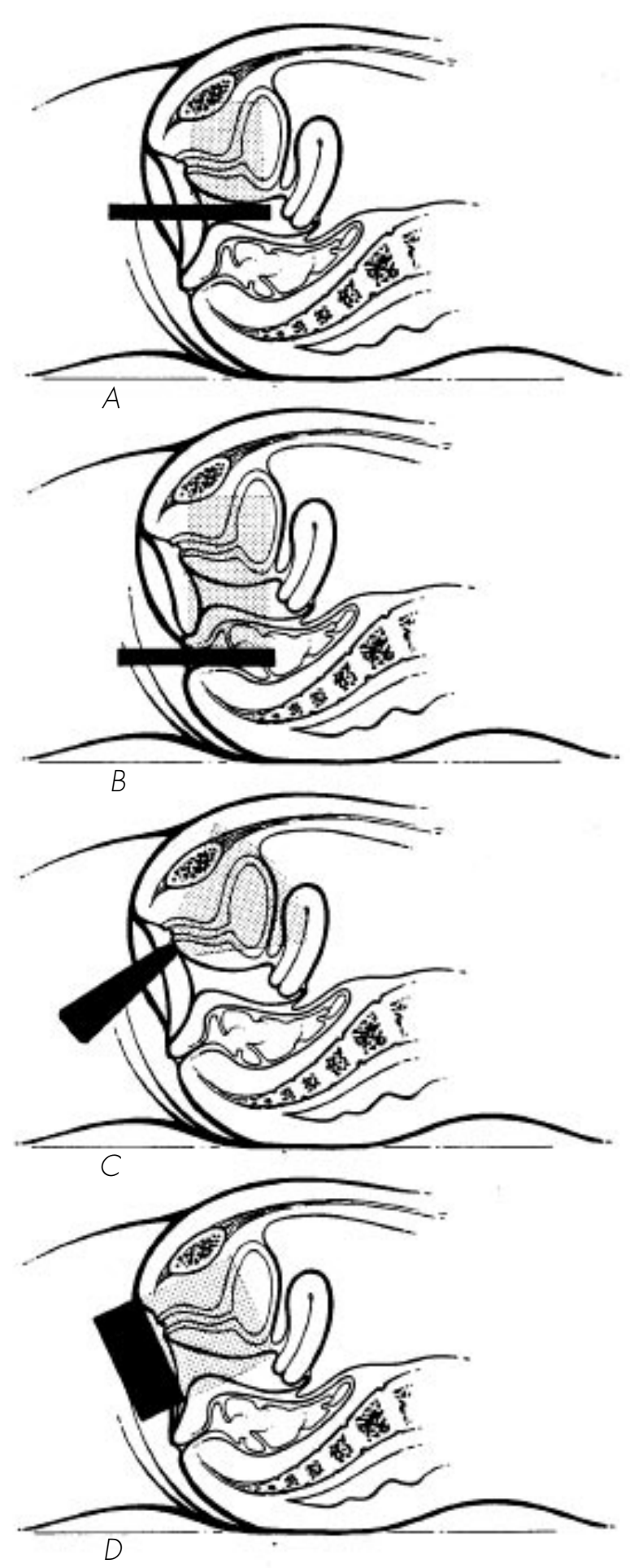

FIGURA 1. Vías de realización de Ecografía para estudio de la Incontinecia urinaria femenina. IA) Transvaginal; 1B) Transrectal; 1C) Introital; 1D) Transperineal o translabial 
La incontinencia urinaria (IU) se define como la pérdida involuntaria de orina y puede ser uretral o extrauretral pudiendo ser esta congénita (ectopia,...), iatrógena o traumática como las fístulas y en algunos casos la disfunción del Tracto Urinario Inferior (TUI) puede llevar al desarrollo de hidronefrosis y fallo renal, como en los pacientes con vejiga neurógena. También el prolapso de órganos pelvianos (POP) severo puede causar angulación del uréter pelviano por las arterias uterinas que lleven a la producción de hidronefrosis.

En general no hay indicación de estudio del TUS en IUF de esfuerzo de urgencia o mixta, pero en las condiciones antes descritas puede ser preciso en (8): (Tabla I)

1. Fístula o uréter ectópico (Sospecha de incontinencia extrauretral).

2. Situaciones de alta presión vesical que pueda repercutir sobre TUS: antecedentes neurógenos, obstrucción crónica con incontinencia o vejigas de baja acomodación al llenado.

3. Excluir hidronefrosis en IU asociada a prolapso de órganos pelvianos (POP) severo.

La ecografía es hoy la técnica de elección a usar en la evaluación del TUS en IU por su bajo coste, gran disponibilidad y falta de radiación. Aporta una adecuada información anatómica, incluyendo detección de malformaciones, tamaño renal, hidronefrosis,

\section{TABLA I. INDICACIONES DE LA ECOGRAFIA ABDOMINAL EN INCONTINENCIA URINARIA FEMENINA.}

1. Determinación del residuo postmiccional.

2. Fístula o uréter ectópico (Sospecha de incontinencia extrauretral).

3. Situaciones de alta presión vesical que pueda repercutir sobre TUS: antecedentes neurógenos, obstrucción crónica con incontinencia o vejigas de baja acomodación al llenado.

4. Excluir hidronefrosis en IU asociada a prolapso de órganos pelvianos (POP) severo. litiasis, neoplasias y repercusión de la disfunción del TUI sobre el TUS. Hay que recordar que no es, sin embargo, una prueba funcional y que el grado de dilatación no se correlaciona con la severidad de la obstrucción. Por el contrario, la ecografía es idónea para seguir el grado de dilatación en el tiempo y/o la respuesta de la misma al tratamiento. La ecografía no es capaz de diferenciar cuando la dilatación se debe a obstrucción, a reflujo o a ambas.

La ecografía se ha convertido en una valiosa exploración para el diagnóstico y seguimiento de la patología del aparato genitourinario. Proporciona una evaluación rápida, poco costosa, no invasiva y no requiere el uso de radiaciones ionizantes.

Se ha intentado usar la ecografía abdominal en el estudio de la IUF. White en 1980, utilizando un transductor lineal, valora el descenso de la unión vésico-uretral (9). Una técnica similar fue utilizada por Bhatia en el año 1990 para determinar la movilidad del cuello vesical antes y después de la uretropexia retropúbica (10). Ambos autores informaron sobre las limitaciones de la técnica debido a la mala visualización de la unión uretrovesical como consecuencia de la sombra producida por el pubis hasta en el $40 \%$ de las pacientes. La ecografía abdominal hipogástrica (o transparietal) hoy sólo se usa en IUF para el estudio de masas pelvianas y la determinación del residuo postmiccional.

\section{ECO DEL TUI Y SUELO PELVIANO EN LA IU FEMENINA}

El aumento de disponibilidad de ECO y RMN ha despertado de nuevo el interés en las técnicas de imagen. La RMN es cara y poco accesible. La ECO se puede usar por 6 vías: abdominal, transperineal (o translabial), introital, transrectal, transvaginal e intrauretral. De ellas la transperineal es la más usada por comodidad al ser la menos invasiva, aunque existen publicaciones a favor $y$ en contra de las diversas vías, según lo que se quiera determinar. La ecografía actualmente permite la evaluación pre y postoperatoria adecuada de la dinámica del cuello vesical y la uretra proximal en la mujer con IUF. Se ha descrito el uso de doppler (34), y el registro de presiones al tiempo (ecourodinámica) (11). Últimalemente ECO transvaginal y translabial en 3D se ha empezado a usar y es previsible que en un futuro próximo adquirirá gran desarrollo.

\section{Ecografía transperineal}

La ecografía transperineal (o translabial) es la técnica más extendida. Permite, mediante la va- 
loración de la relación entre la uretra y el pubis (7), evaluar la posición del cuello vesical, los cambios anatómicos, el desplazamiento del cuello vesical con la realización de la maniobra de Valsalva, la presencia o no de cistocele, y si existe embudización de la uretra con la consecuente pérdida de orina (8). La ecografía transperineal se ha comparado con la CUMS y la cistografía con cadena (12) y se encontró una buena correlación en la determinación de la posición y movilidad del cuello vesical, ángulo vésicouretral posterior, inclinación uretral, distancia sínfisis del pubis a cuello vesical y ángulo de rotación de la uretra (Tabla II). La especificidad, sensibilidad y acuerdo interobservadores también fueron comparables para los dos métodos. Por ello todos los autores parecen preferir la ECO. Sólo la embudización del cuello parece ser demostrada mejor y más fácilmente por la cistografía en bipedestación que por la ecografía realizada en posición supino (13).

- Detalles técnicos: En ella se obtiene un corte mediosagital colocando el transductor de $3,5-7 \mathrm{MHz} \mathrm{MHz}$ en el periné cubierto con una funda de látex, y de $4-8 \mathrm{~cm}$ de tamaño. Los de mayor tamaño son preferibles si se quieren explorar los 3 compartimentos La imagen obtenida incluye sínfisis del pubis anteriormente, uretra y cuello vesical, vagina, cerviz uterino, recto y canal anal. Tras la unión anorectal hay una imagen hiperecogénica que corresponde a la porción central del elevador. A veces se ve el cul de sac peritoneal lleno de líquido, grasa o asas de intestino

TABLA II. UTILIDAD DE LA ECOGRAFÍA TRANSPERINEAL EN EL ESTUDIO DE LA INCONTINENCIA URINARIA FEMENINA.

1. Posición y movilidad del cuello vesical y uretra proximal

2. Documentar la Incontinencia Urinaria Femenina de Esfuerzo

3. Medida del grosor de la pared vesical

4. Actividad del elevador del ano

5. Cuantificación del Prolapso de Órganos Pelvianos (POP)

6. Visualización de las mallas

7. Otros hallazgos (Divertículos uretrales, tumores vesicales, ...) con contenido y en movimiento. Cortes parasagitales o transversales pueden añadir información (en la evaluación del músculo puborectalis) (8).

Para proteger el transductor se deben evitar las fundas empolvadas pues deterioran la imagen obtenida. La ecografía puede hacerse en decúbito lateral con las rodillas flexionadas o de pie. La repleción vesical deberá especificarse. Para algunas aplicaciones es preferible orinar primero. La ampolla llena de heces puede presentar problemas de visualización. Al colocar el transductor separar los labios puede mejorar la calidad de imagen. La imagen obtenida da información similar o mejor a CUMS lateral o fluoroscopia (8).

La cuantificación de la posición de los órganos o la medición de los ángulos se lleva a cabo en un sistema de coordenadas formado por:

- Líneas paralelas y verticales al eje del transductor con referencia al borde inferior de a sínfisi del pubis o

- El eje central de la sínfisis del pubis y la línea vertical a este eje.

Las condiciones en que se realiza la exploración (vejiga llena o no, con sonda, de pie o sentada, vía de exploración,..) deben reseñarse, pues no hay un estándar universal $(16,8,18)$.

\section{Posición y movilidad del cuello vesical y uretra proximal:}

La posición y movilidad del cuello vesical y uretra proximal pueden evaluarse con un elevado grado de fiabilidad. Los puntos de referencia inmóviles son el eje central de la sínfisis del pubis (14) o su margen inferior (15). El eje central de la sínfisis puede ser más difícil de ver si el disco articular está calcificado. Las imágenes se pueden tomar en distintas posiciones y con vejiga llena o vacía, pero la vejiga llena puede limitar la movilidad de la vejiga y ocultar la presencia de POP (16). En bipedestación la vejiga está en posición más baja que en decúbito, pero el descenso con la maniobra de Valsalva es similar (17). Es importante no ejercer Presión excesiva con el trasnductor que limite el descenso de la vejiga. La uretra de la paciente con hipermovilidad uretral sale de una posición en reposo más baja y desciende más con el esfuerzo, que la de la paciente continente.

En el estudio se realizan medidas en reposo y con Valsalva. La diferencia entre ellas da un valor numérico que refleja el descenso del cuello de la vejiga. Además en el Valsalva se ve si hay rotación de la uretra proximal en dirección posteroinferior. El 
grado de rotación (ángulo rotacional) que se puede medir desde distintos sitios: comparando el ángulo de inclinación entre la uretra proximal móvil y la uretra distal, llamado ángulo beta (18), obtenido por visualización del acodamiento uretral (urethral knee), que es el punto donde la uretra entra en el diafragma urogenital (Figura 2). En él, esta porción distal de la uretra está rodeada por dos arcos de músculo estriado: el compresor uretral y el esfínter uretrovaginal. Algunos miden el ángulo retrovesical o uretrovesical posterior que es el formado entre la uretra proximal y el trígono (19), otros determinan el ángulo alfa entre el eje central de la sínfisis del pubis y la línea que va desde el borde inferior de la sínfisis al cuello de la vejiga (20) (Figura 3). De todos los parámetros ecográficos de hipermovilidad, parece ser que el descenso del cuello vesical es el que tiene mayor asociación con la incontinencia de esfuerzo urodinámica (21). La reproductibilidad de este parámetro dinámico ha sido establecida como excelente para múltiples maniobras de Valsalva en las mismas pacientes y entre diferentes ecografistas (22).

No se ha definido un valor de descenso normal del cuello vesical, aunque se han propuesto valores de corte de 20 y $25 \mathrm{~mm}$ para definir hipermovilidad. El grado de llenado vesical, la posición de la paciente, y la catereterización pueden influir en las medidas. En nulíparas puede ser difícil obtener una maniobra de Valsalva efectiva, por lo que establecer un valor basal normal no ha sido fácil y en la literatura se recogen descensos del cuello vesical de 5 a

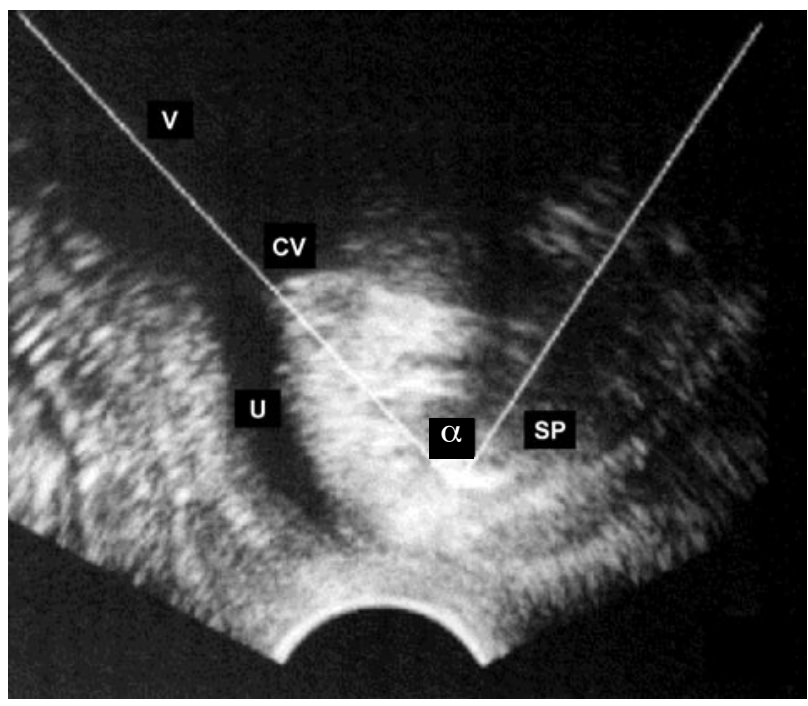

FIGURA 2. El ángulo alfa $(\alpha)$ entre la línea que une cuello con el borde inferior de la sinfisis y la línea media de la sínfisis. U: uretra; SP: Sínfisis del pubis; V: vejiga; CV Cuello vesical
$15 \mathrm{~mm}(23,24)$. Diferencias metodológicas podrían explicar éstas divergencias. No existe tampoco una definición de cómo realizar una maniobra de Valsalva estándar.

En mujeres con IUE se ha descrito la embudización del cuello vesical con el Valsalva (12), como demostración de la hipermovilidad de la uretra, a consecuencia del defecto de soporte extrínseco $(12,10)$, pero también se ha visto que hay embudización hasta en el $30 \%$ de las mujeres sanas en reposo, con vejiga llena (25) y en mujeres conincontinencia de urgencia (26). La embudización se asocia con frecuencia con fugas, pero igual que en radiología no es un criterio diagnóstico de IUF. La embudización marcada se asocia a presiones uretrales de cierre bajas, sugiriendo la presencia de Insuficiencia intrínseca esfinteriana.

El hallazgo más frecuente en casos de hipermovilidad del cuello vesical es el descenso y rotación del meato interno, que se da por la rotación de la uretra proximal y el trígono en dirección posteroinferior. El ángulo retrovesical normal de 90-120 aumenta hasta 160-180, lo cual a menudo se asocia a embudización, pero no siempre, como puede verse en pacientes con cistocele y continentes (8). Se ha sugerido que esta situación es lo que diferencia el defecto central de los laterales de la fascia endopélvica. El acodamiento de la uretra en estos casos puede producir dificultad miccional que empeora con el esfuerzo, con la repleción marcada vesical y con la protrusión

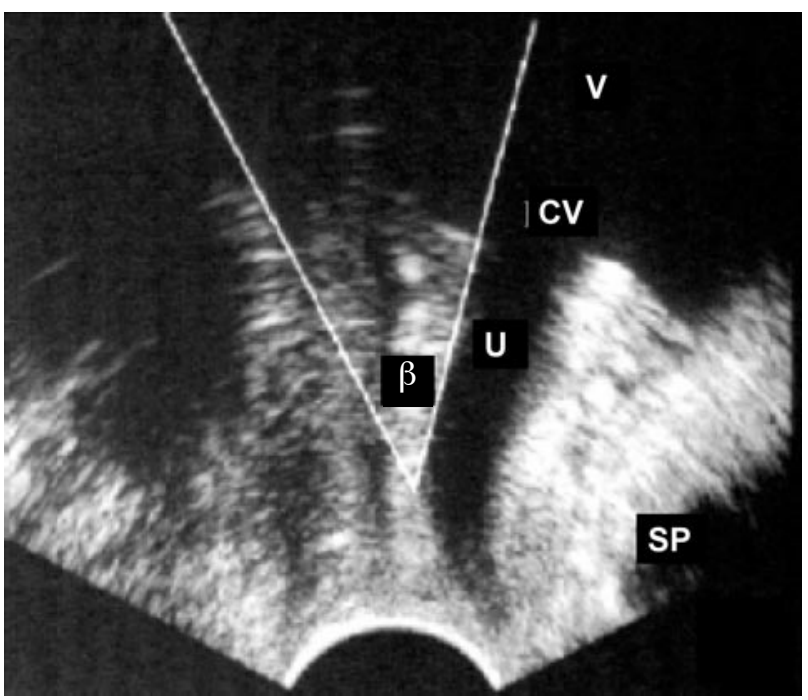

FIGURA 3. El ángulo beta $(\beta)$ entre las porciones proximal (móvill y distal (fija) de la uretra y el codo uretral visualizados. U: uretra; CV: cuello vesical; SP: sínfisis del pubis; V: vejiga. 
del prolapso, pudiendo llegar a la retención aguda de orina. Es sabido que tras el tratamiento con éxito del prolapso puede ponerse de manifiesto una IUF de esfuerzo oculta por la presencia del prolapso.

\section{Documentar la IUE:}

La ECO se puede usar como alterativa al test de esfuerzo para demostrar la IUE durante las maniobras de provocación. Para ello se han usado diversas técnicas:

- Se ha intentado hacer visible la fuga en ECO con medios de contraste sonográficos con microburbujas como el Echovist, pero es caro y requiere cateterización (27).

- Doppler color: la correlación entre los hallazgos fluoroscópicos y sonográficos fue muy buena y no dependió de la presencia de sondas (28). El Doppler color puede también facilitar la documentación de la Presión en el Punto de Fuga (Leak Point Pressure), aunque este parámetro urodinámico es cada vez menos usado.

\section{Medida del grosor de la pared vesical:}

Puede determinarse vía transvaginal o translabial. Se toman 3 medidas del grosor de la pared en cara anterior, trígono y cúpula. A mayor grosor de la pared, mayor posibilidad de padecer una vejiga hiperactiva (6). Si es mayor de $5 \mathrm{~mm}$ se asocia a detrusor hiperactivo sobre todo si se asocia a clínica de hiperactividad. Se están desarrollando software y hardware para automatizar las medidas y cálculos de medidas, tanto en varones como en mujeres, pero su uso en clínica todavía no está extendido ni validado.

\section{Actividad del elevador del ano:}

Los ultrasonidos perineales se han usado para demostrar y cuantificar la actividad muscular del suelo de la pelvis en mujeres continentes e incontinentes y para estudiar los cambios antes y después del parto. La elevación craneal de los órganos pelvianos en el corte sagital en línea media revela la contracción del elevador. El desplazamiento del meato uretral interno, desde su posición en reposo, se mide con relación la borde postero-inferior del pubis. Además se puede usar la ecografía para el biofeedback.

Por otra parte la ecografía ha ayudado a validar el concepto del "bloqueo perineal" (Knack). Este consiste en la contracción del elevador del ano inmediatamente antes del aumento de Presión intraabdominal, y se enseña a las pacientes al tiempo que se les instruye en los ejercicios del suelo de la pelvis y sirve para evitar o disminuir la intensidad de la fuga en el momento del esfuerzo (tos, risa, Vasalva...) (29).

\section{Cuantificación del Prolapso de Órganos Pelvianos (POP):}

La ECO translabial puede demostrar el POP. El borde inferior de la sínfisis del pubis sirve como referencia para medir el máximo descenso de vejiga, útero, fondo de saco y ampolla rectal en Valsalva. Recientemente se ha encontrado una buena correlación con el sistema desarrollado por la ICS para la evaluación del POP (POP-Q) para los compartimentos anterior y medio, y peor para el compartimento posterior. Algunas desventajas de la ecografía serían la incompleta visualización del cérvix o cúpula en presencia de un gran rectocele y la posible subestimación del prolapso por la presión del transductor. EL POP severo (procidencia) impide la realización de la ECO (30).

\section{Visualización de mallas}

La ECO permite además visualizar los materiales sintéticos detrás de la uretra o trígono, ya sean mallas o inyectables. Ello junto a su capacidad de realizar estudios dinámicos ha permitido estudiar las diferencias antes y después de la cirugía y el mecanismo por el cual se produce la continencia y las disfunciones tras la cirugía de la IUF. Se ha podido así ver que la sobreelevación del cuello en la colposuspensión es innecesaria para la cura de la IUE y puede asociarse al desarrollo de disfunción miccional (31).

La ecografía ha contribuido significativamente en la investigación de nuevos tratamientos quirúrgicos, ha ayudado a clarificar como actúan (32) (acodamiento uretral o compresión dinámica) y parece en esto superior a la RMN. La evaluación de la movilidad del cuello vesical antes del implante de un cabestrillo suburetral tipo TVT, puede predecir el éxito quirúrgico, algo lógico si pensamos que la compresión dinámica depende de la diferencia relativa de movilidad del implante y de los tejidos de la paciente (33).

\section{Otros hallazgos:}

Se pueden detectar con ECO trasnperineal divertículos vesicales, uretrales, litiasis, Tumores vesicales,.... La ECO 3-D, abre nuevas posibilidades de observar la anatomía funcional y examinar estructuras musculares y faciales del suelo pelviano fácilmente ayudándonos a identificar mejor los defectos y preparar cirugías selectivas para ellos (34). 


\section{DISCUSIÓN}

El diagnóstico por imagen de la IU, en general, y de la IUF, en particular, pronto ganó interés históricamente. La Cistografía Miccional Seriada (CUMS) fue la técnica de imagen inicial. Posteriormente, tras ser muy discutida su utilidad en el manejo de la incontinencia, los clínicos que han trabajado en urología femenina se han enfocado sólo a los parámetros urodinámicos de la cistomanometría y perfil uretral. El problema de la radiología convencional es que no se pudo demostrar relación entre anatomía y función, ni diferencias entre continentes e incontinentes que fueran constantes (8).

El aumento de disponibilidad de ECO y RMN ha despertado de nuevo el interés en las técnicas de imagen. La posibilidad de realizar exploraciones dinámicas de imagen también ha contribuido a ello. La RMN es cara y poco accesible. La ECO se puede usar por 5 vías: abdominal, transperineal (o translabial), introital, transrectal, transvaginal. De ellas la perineal es la más usada por comodidad por ser la menos invasiva, aunque existen publicaciones a favor y en contra de las diversas vías, según lo que se quiera determinar y si se quiere usar doppler, o registrar presiones al tiempo (ecourodinámica). Últimalemente ECO transvaginal y translabial en 3D se ha empezado a usar y es previsible que en un futuro próximo adquirirá gran desarrollo (8).

La ecografía, ha sustituido a la radiología convencional por su inmediatez, bajo coste y nulos efectos secundarios. La TAC nunca ocupó un lugar importante, pero en los últimos años la RMN, por su capacidad de obtener rápidamente imágenes dinámicas de toda la pelvis y sus diferentes tejidos, está apareciendo con fuerza en el estudio de la patología

TABLA III. RECOMENDACIONES DE LA $3^{\underline{a}}$ INTERNATIONAL CONSULTATION ON INCONTINENCE.

1. Ultrasonidos NO SE RECOMIENDAN EN LA EVALUACIÓN PRIMARIA de las pacientes con IU y/o POP (Nivel3 de evidencia, Grado de recomendación $\mathrm{C}$ )

2. Ultrasonidos ES UNA PRUEBA OPCIONAL EN LA EVALUACIÓN DE PACIENTES CON IU Y/O POP RECURRENTES O COMPLEJAS

(Nivel de evidencia 3, Grado de recomendación C). funcional, aunque su lugar final, en la clínica, no está claro.

La razón para el estudio por técnicas de imagen del TUI en IU es la supuesta relación morfología/función y particularmente el papel causal que se le atribuye a la presencia de hipermovilidad uretral en la Incontinencia Urinaria de Esfuerzo Femenina (IUEF). Con el tiempo se hizo evidente que reparación anatómica y curación no iban necesariamente pareja y sólo la llegada del Ecografía ha hecho que se renueve el interés por usar técnicas de imagen.

El problema fundamental de la ecografía, para la universalización de su uso en el estudio de la IUF, es que se han publicado resultados de su uso por 5 vías (abdominal, transperineal (o translabial), introital, transrectal, transvaginal); con diferentes tipos de transductores; diferentes mediciones lángulos y distancias entre diferentes estructuras) y con una falta de estandarización en las condiciones en que se debe usar este estudio. Todo ello ha creado mucha literatura, difícil de comparar, y que no permite una adecuada validación de la técnica. Hay todavía escasez de estudios que comparen datos en pacientes continentes e incontinentes, rangos de valores normales en diferentes edades y cual es la influencia de la paridad, estado hormonal,... En cualquier caso la ecografía no es hoy una técnica de realización imprescindible en el estudio de la IUF (Tabla III) (8).

De las posibles vías de estudio la ecografía trasnperineal o translabial es la más usada por comodidad al ser la menos invasiva. Pero existen publicaciones a favor y en contra de las diversas vías, según lo que se quiera determinar y si se quiere usar doppler, o registrar presiones al tiempo (Ecourodinámica). Últimamente la ECO transvaginal y translabial en 3D se ha empezado a usar y es previsible que en un futuro próximo adquirirá gran desarrollo (34). La ECO introital, que usa ECO endocavitaria, con transductores sectoriales pequeños con resolución más alta de la uretra y tejidos parauretrales no permite la visualización de los 3 compartimentos y dificulta la cuantificación de datos al quedar la sínfisis fuera de campo visualizado. De todas formas, a pesar de lo que hoy sabemos, y ante las diversas vías de acceso y variables a medir propuestas, se necesitan más estudios para alcanzar un acuerdo universal y analizar su valor real.

La ecografía se puede usar como alternativa a las técnicas radiológicas convencionales para la evaluación preoperatoria y postoperatoria de las mujeres con incontinecia $(8,14)$. La movilidad del cuello vesical se puede demostrar con ultrasonidos por vía vaginal o perineal usando la sínfisis como 


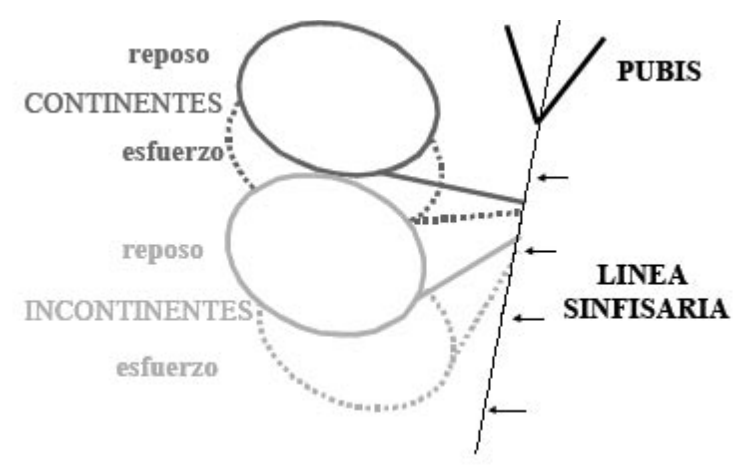

FIGURA 4. Comportamiento del tercio proximal de la uretra al esfuerzo en mujeres continentes e incontinentes (6).

punto de referencia fijo (20). (Figura 4) Además, la ecografía transperineal permite ver y medir diversas referencias, siendo posible efectuar una observación dinámica de la disfunción de la mujer incontinente: aumento de la movilidad del cuello, disminución de la angulación de la uretra (35).

\section{CONCLUSIONES}

Los ultrasonidos y en particular la ECO transperineal o translabial está en proceso de convertirse un método diagnóstico estándar en uroginecología. A ello contribuye su amplia disponibilidad, estandarización de los parámetros, posibilidad de evaluar no sólo la vejiga, sino también el elevador del ano - los Prolapsos de órganos pelvianos (POP). Permite obtener, de forma no invasiva datos pretratamiento y postratamiento. Por ahora podemos decir que:

- La ecografía es ampliamente usada en mujeres con IU y prolapso. Debido a su bajo coste, disponibilidad, inmediatez, falta de efectos secundarios es el método de imagen más conveniente actualmente

- La ecografía transperineal es la más usada de las diversas vías de evaluación en IUF, por su baja invasividad y poca distorsión de los tejidos

- La exploración del suelo de la pelvis con ecografía permite determinar la posición y movilidad de los órganos pelvianos en reposo, con la tos y evaluar la continencia urinaria y el prolapso. Se puede medir la rotación de la uretra comparando los ángulos de inclinación en reposo y con Valsalva, el ángulo retrovesical en ambas situaciones, la aparición de embudización con el Valsalva.

- Los datos de la ecografía del descenso del cuello vesical, embudización y fuga de orina se correlacionan bien con los hallazgos radiológicos.
- La evaluación del POP se correlaciona bien con el sistema POP-Q de la ICS.

- Pueden usarse la ECO para el biofeedback pues pone de manifiesto la contracción del elevador del ano y su efecto. Se correlaciona bien con los datos de perineometría y evaluación digital.

- El Doppler color transperineal se puede usar para poner de manifiesto la IUE Femenina.

- ECO es el método de elección en la identificación de sling suburetral y otros implantes ecogénicos (como los medios inyectables).

- La ECO 3D permite ahora obtener imágenes en el plano axial tales como el parauretral/paravaginal.

\section{BIBLIOGRAFÍA y LECTURAS RECOMENDADAS (*lectura de interés $y^{* *}$ lectura fundamental)}

1. KOHOM, E.L.; SCIOSCIA, A.L.; JEANTY, P. y cols.: "Ultrasound cystourethrography by perineal scanning for the assessment of female stress urinary incontinence". Obstet. Gynecol., 68: 269, 1986.

2. BERGMAN, A.; McKENZIE, C.J.; RICHMOND, J. y cols.: "Transrectal ultrasound versus cystography in the evaluation of anatomical stress urinary incontinence." Br. J. Urol., 62: 228, 1998.

3. FERNÁNDEZ, I.; RUIZ, J.L.; LLORENTE, C. y cols.: "Técnica de la ecografía transrectal en la incontinencia urinaria de esfuerzo femenina". Arch. Esp. Urol., 48: 185, 1995.

4. QUINN, M.J.; BEYNON, J.; MORTENSEN, N.J. y cols.: "Transvaginal endosonography: a new method to study the anatomy of the lower urinary tract in urinary stress incontinence". Br. J. Uro1., 62: 414, 1988.

5. KOEBL, H.; BERNASCHEK, G.; DEUTIGER, J.: "Assesment of female urinary incontinence by introital sonography". J. Clin. Ultrasound, 18: 370, 1990.

*6. CASSADÓ GARRIGA, J.: "Estudio ecográfico uretrovesical introital comparativo entre mujeres con incontinencia urinaria de esfuerzo por hipermovilidad uretral y mujeres continentes. Valoración de variables ecográficas discriminatorias". Tesis doctoral .Universidad Autónoma de Barcelona. Barcelona 2001. Disponible en www. tdx.cesca.es/TESIS_UAB/ AVAILABLE/TDX1010102-142725//jcg3de3.pdf

7. FERNÁNDEZ GONZÁLEZ, I.; MAYAYO DEHESA, T.; BERENGUER SÁNCHEZ, A.: "La ecografía en el diagnostico de la incontinencia urinaria". Clínicas Urológicas de la complutense, 8: 117, 2000. 
**8. TUBARO, A.; ARTIBANI, W.; BARTAM, C. y cols.: "Committee 9". Incontinence 3rd International Consultation on Incontinence- Ed.: Abrams, P., Cardozo L, Khoury, S., Wein, A. 707, 2005.

9. WHITE, R.D.; McQUOWN, D.; McARTHY, T.A. y cols.: "Real time ultrasonography in the evaluation of urinary stress incontinence". Am. J. Obstet. Gynecol., 138: 235, 1980.

10. BATHIA, N.N.; OSTERGARD, D.R.; McQUOWN, D.: "Ultrasonography in urinary incontinence". Urology, 29, 1990.

11. VIERHOUT, M.E.; HOL, M.: "Vaginal ultrasound studies before and after successful colposuspension and in continent controls". Act. Obstet. Gynecol. Scand., 77: 101, 1998.

*12. HUANG, W.C.; YANG, J.M.: "Bladder neck funneling on ultrasound cystourethrography in primary stress urinary incontinence: a sign associated with urethral hypermobility and intrinsic sphincter deficiency". Urology, 61: 936, 2003.

13. DIETZ, H.P.; CLARKE, B.: "The influence of posture on the perineal ultrasound imaging parameters". International Urogynecology Journal, 12: 104, 2001.

*14. SCHAER, G.N.; KOECHLI, O.R.; SCHUESSLER, B. y cols.: "Perineal ultrasound for evaluating the bladder neck in urinary stress incontinence". Obstet. Ginecol., 85: 220, 1995.

*15. DIETZ, H.P.; WILSON, P.D.: "Anatomica1 assessment of the bladder outlet and proximal urethra using ultrasound and videocystourethrography". Neurourology and Urodinamycs, 15: 363, 1996.

16. DIETZ, H.P.; WILSON, P.D.: "The influence of bladder volume in the position and mobility of the urethrovesical junction". Int. Umgynecol. J., 10: 3, 1999.

17. DIETZ, H.P.; CLARKE, B.: "The influence of posture on the perineal ultrasound imaging parameters". International Urogynecology Journal, 12: 104, 2001.

*18. VIERHOUT, M.E.; HOL, M.: "The knee of the urethra: its possible role in the female continence mechanism". Urogyn. Int. J., 8: 81, 1994.

19. ALPER, T.; CETINKAYA, M.; OKUTGCN, S. y cols.: "Evaluation of urethrovesical angle by ultrasound in women with and without urinary stress incontinente". International Urogynecology Journal, 12: 308, 2001.

20. MOURITSEN, L.; RASMUSSEN, A.: "Bladder neck mobility evaluated by vaginal ultrasonography". Br. J. Urol., 71: 166, 1993.

21. DIETZ, H.P.; CLARKE, B.; HERBISON, P.: "Bladder neck mobility and urethral closure pressure as predictors of genuine stress incontinence". International Urogynecology Journal, 13: 289, 2002.
*22. DIETZ, H.P.; ELDRIDGE, A.; GRACE, M. y cols.: "Test- retest reliability of the ultrasound assessment of bladder neck mobility". International Urogynecology Journal, 14: 57, 2003.

23. REED, H.; WATERFIELD, A.; FREEMAN, R.M. y cols.: "Bladder neck mobility in continent nulliparous women: normal references". Int. Urogynecol. J., 13: 4, 2002.

24. PESCHERS, U.M.; FANGER, O.; SCHAER, O.N. y cols.: "Bladder neck mobility in continent nulliparous women”. BJOG, 108: 320, 2001.

25. VERSI, E.: "The significance of an open bladder neck in women". Br. J. Urol., 68: 42, 1991.

*26. SCHAER, G.N.; PERUCCHINI, D.; MUNZ, E. y cols.: "Sonographic evaluation of the bladder neck in continent and stress-incontinent women". Obstet. Gynecol., 93: 412, 1999.

27. SCHAER, O.N.; KOECHLI, O.R.; SCHUESSLER, B. y cols.: "Usefulness of ultrasound contrast medium in perineal sonography for visualization of bladder neck funelling-first observations". Urology, 47: 452, 1996.

28. DIETZ, H.P.; CLARKE, B.: "Translabial color Doppler urodynamics". International Urogynecology J., 12: 304, 2001.

29. MILLER, J.M.; PERUCCHINI, D.; CARCHIDI, L.T. y cols.: "Pelvic floor muscle contraction during a cough and decreased vesical neck mobility". Obstetrics \& Gynecology, 97: 255, 2001.

30. DIETZ, H.P.; HAYLEN, B.T.; BOOME, J.: "Ultrasound in the quantification of female pelvis organ prolapse ultrasound". Obstetrics and Gynecology, 18: 511, 2001.

31. BOMBIERI, L.; FREEMAN, R.M.: "Do bladder neck position and amount of elevation influence the surgical outcome of colposuspension?" Neurourol Urodyn, 18: 316, 1999.

32. LO, T.S.; HORNG, S.G.; LIANG, C.C. y cols.: "Ultrasonographic assessment of mid-urethra tape at three years of follow-up after tension-free vaginal tape procedure (TVT)". Urology, 63: 671, 2004.

33. FRITEL, X.; ZABAK, K.; PIGNE, A.; BENIFLA, J.: "Predictive value of the uretra mobility before sub-urethral tape procedure for stress urinary incontinence in women". Proceedings ICS, 190, 2002.

34. KULLHAR, V.; CARDOZO, L.: "Three Dimensional ultrasound in Urogynecology". Merz E. editor. 3-D Ultrasound in Obstetrics and Gynecology. Philadelphia: Lippincot, Williams and Wilkins Healthcare, 65, 1998.

35. PREGAZZI, R.; SARTOREA, A.; BORTOLIB, P. y cols.: "Perineal ultrasound evaluation of urethral angle and bladder neck mobility in women with stress urinary incontinence". BJOG, 109: $821,2002$. 